\title{
Rosácea oculocutánea en un niño
}

\author{
Ocular and cutaneous rosacea in a child
}

\author{
Dra. María C. Di Matteo , Dra. Paola C. Stefano ${ }^{a}$ Dra. Ana Cirio ${ }^{a}$, Dra. Beatriz López ${ }^{b}$, Dra. María Centeno ${ }^{c}$ \\ Dra. Marcela Bocian ${ }^{a}$ y Dra. Andrea B. Cervinia
}

\begin{abstract}
RESUMEN
La rosácea es una dermatosis crónica que se manifiesta clínicamente con eritema, telangiectasias, pápulas y pústulas en la parte central de la cara. Afecta, sobre todo, a los adultos y, en raras ocasiones, a los niños. La rosácea puede también presentar afección ocular y preceder, aparecer en forma simultánea o con posterioridad a las lesiones cutáneas; es mayor el riesgo de complicaciones oculares en los niños. La baja prevalencia de esta patología en la infancia origina demoras en el diagnóstico. Se presenta a un paciente de 1 año de edad con rosácea ocular y cutánea que desarrolló opacidades corneales con compromiso de la agudeza visual.

Se destaca la importancia del diagnóstico y del tratamiento precoz para evitar el desarrollo de secuelas.

Palabras clave: rosácea ocular, niño.
\end{abstract}

\begin{abstract}
Rosacea is a chronic skin disease characterized by erythema, telangiectasia, papules and pustules in the central facial region. It most often affects adults and is rare in children. Rosacea can also present ocular involvement. Symptoms can precede cutaneous findings, appear simultaneously or after them, with a higher risk of ocular complications in children. Because of low prevalence of rosacea in childhood, the diagnosis is frequently delayed.

We report a 1-year-old boy with ocular and cutaneous rosacea who developed corneal opacities and visual impairment.

Early diagnosis and treatment is considerable to avoid sequels. Key words: ocular rosacea, child.
\end{abstract}

http: / / dx.doi.org/10.5546/ aap.2019.e170

Cómo citar: Di Matteo MC, Stefano PC, Cirio A, López B, et al. Rosácea oculocutánea en un niño. Arch Argent Pediatr 2019;117(2):e170-e172.

a. Servicio de Dermatología, Hospital de Pediatría "Prof. Dr. Juan P. Garrahan", Buenos Aires, Argentina.

b. Servicio de Oftalmología, Hospital de Pediatría "Prof. Dr. Juan P. Garrahan", Buenos Aires, Argentina.

c. Servicio de Anatomía Patológica, Hospital de Pediatría

"Prof. Dr. Juan P. Garrahan", Buenos Aires, Argentina.

Correspondencia:

Dra. María C. Di Matteo: celestedimatteo@gmail.com

Financiamiento: Ninguno.

Conflicto de intereses: Ninguno que declarar.

Recibido: 2-6-2018

Aceptado: 31-10-2018

\section{INTRODUCCIÓN}

La rosácea es una afección inflamatoria crónica de la piel, de causa desconocida, que afecta, principalmente, las áreas convexas de la cara. Es más frecuente en las personas con fototipo bajo (I-II) de la piel y suele manifestarse entre la tercera y la quinta década de la vida. ${ }^{1} \mathrm{Si}$ bien existen reportes en niños, la prevalencia en este grupo etario es desconocida. ${ }^{1-3}$ Se manifiesta clínicamente por flushing, eritema centrofacial y telangiectasias, asociadas o no a la presencia de pápulas, pústulas, hipertrofia glandular y/o fibrosis (fimas). ${ }^{4}$ El compromiso ocular ocurre en alrededor del $30 \%$ al $50 \%$ de los adultos afectados y se manifiesta como blefaritis, chalazión recidivante, queratoconjuntivitis, hiperemia conjuntival, neovascularización y úlceras corneales, entre otros. ${ }^{5}$ Puede manifestarse antes, durante o después de las lesiones cutáneas. ${ }^{6}$

\section{CASO CLÍNICO}

Paciente de sexo masculino de 1 año de edad que consultó al Servicio de Dermatología por pápulas eritematosas pruriginosas en las mejillas y en el puente nasal de un mes de evolución, que fueron interpretadas, inicialmente, como prurigo y, luego, como dermatitis periorificial. Cuatro meses más tarde, comenzó con episodios de queratoconjuntivitis y chalazión recidivante con evolución tórpida, y desarrolló abscesos y úlceras corneales que requirieron antibióticos tópicos (eritromicina, azitromicina, cefalotina, ceftazidima, vancomicina) y orales (eritromicina, clindamicina) e implante de amnios. Debido al compromiso ocular y a la persistencia de lesiones cutáneas recurrentes, fue derivado nuevamente al Servicio de Dermatología con sospecha de rosácea oculocutánea.

Al momento del examen físico, presentaba pápulas eritematosas infiltradas en las mejillas y en el mentón (Figura 1). La madre no refería antecedentes de eritema facial transitorio y no se observaban telangiectasias ni otras lesiones en el resto del tegumento. Como diagnósticos diferenciales, se plantearon rosácea oculocutánea, demodecidosis, sarcoidosis y/o 
enfermedad granulomatosa crónica.

Se realizó una biopsia cutánea y se observaron, en todo el espesor de la dermis, numerosos vasos telangiectásicos y moderado infiltrado inflamatorio linfohistiocitario y neutrofílico que rodeaba las estructuras vasculares y folículos pilosos dilatados. De acuerdo con los hallazgos clínicos e histopatológicos, se confirmó el diagnóstico de rosácea.

Inició el tratamiento con eritromicina a razón de $40 \mathrm{mg} / \mathrm{kg} /$ día por vía oral, metronidazol $1 \%$ tópico y fotoprotección durante 6 meses, y se observó la desaparición de las lesiones cutáneas sin recurrencias (Figura 2). En cuanto al compromiso ocular, si bien se evidenció una franca mejoría de sus lesiones, presentó como secuelas la disminución de la agudeza visual y un leucoma bilateral (Figura 3).

\section{DISCUSIÓN}

La rosácea es una enfermedad inflamatoria crónica de la piel, de causa desconocida, de inicio entre la tercera y la quinta década de la vida. Predomina en las mujeres, de fototipo I y II de la escala de Fitzpatrick y se manifiesta clínicamente por flushing, eritema centrofacial y telangiectasias, asociadas o no a la presencia de pápulas, pústulas, hipertrofia glandular y/o fibrosis (fimas). ${ }^{1}$

Pueden definirse cuatro subtipos de rosácea según los hallazgos clínicos predominantes: eritematotelangiectásica, papulopustulosa,

Figura 1. Pápulas eritematosas en las mejillas, en el puente nasal, en el labio superior y en el mentón al momento del diagnóstico de rosácea cutánea

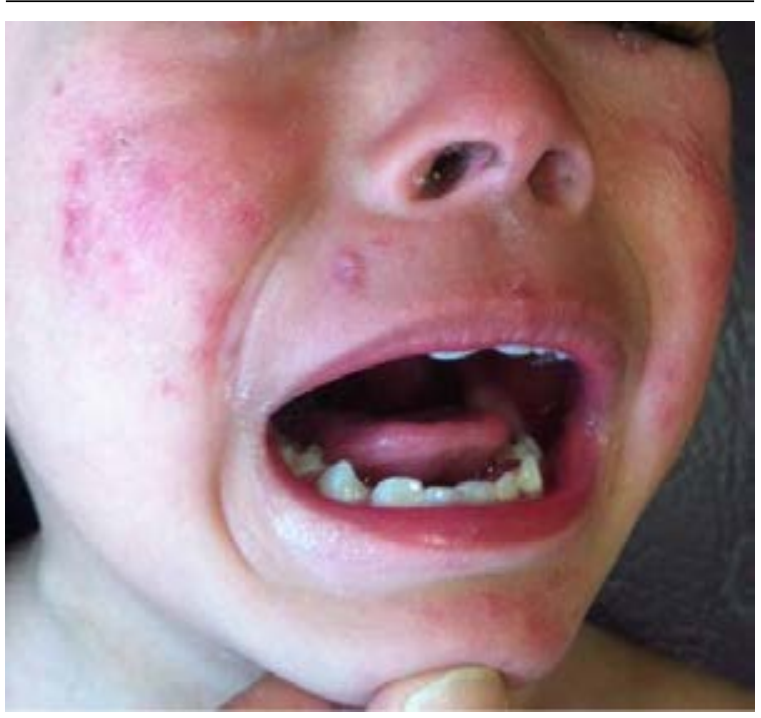

glandular hiperplásica / fimatosa y ocular. Recientemente, la rosácea infantil fue incluida dentro de las formas especiales en la clasificación elaborada por el Grupo Ibero-Latinoamericano de Estudio de la Rosácea (GILER). ${ }^{7}$

La prevalencia de la afectación ocular varía del $30 \%$ al $50 \%$ en los adultos. ${ }^{5}$ Si bien existen reportes en niños, la prevalencia en este grupo etario es desconocida..$^{1-3} \mathrm{En}$, aproximadamente, el $20 \%$ de los casos, los síntomas oculares preceden a los hallazgos cutáneos, y no existe correlación entre la gravedad del compromiso ocular y la rosácea cutánea. ${ }^{6}$ Los hallazgos oculares más frecuentes corresponden a blefaritis, queratoconjuntivitis, chalazión recidivante, hiperemia conjuntival, neovascularización y úlceras corneales. La afectación ocular en los niños se asocia con un porcentaje significativamente mayor de complicaciones (opacidades corneales, disminución de la agudeza visual) que en la población adulta. ${ }^{8}$ Las secuelas cicatrizales pueden comprometer la agudeza visual, por lo que es importante la sospecha precoz para arribar

FIgURA 2. Ausencia de lesiones cutáneas luego del tratamiento con eritromicina y metronidazol durante 6 meses

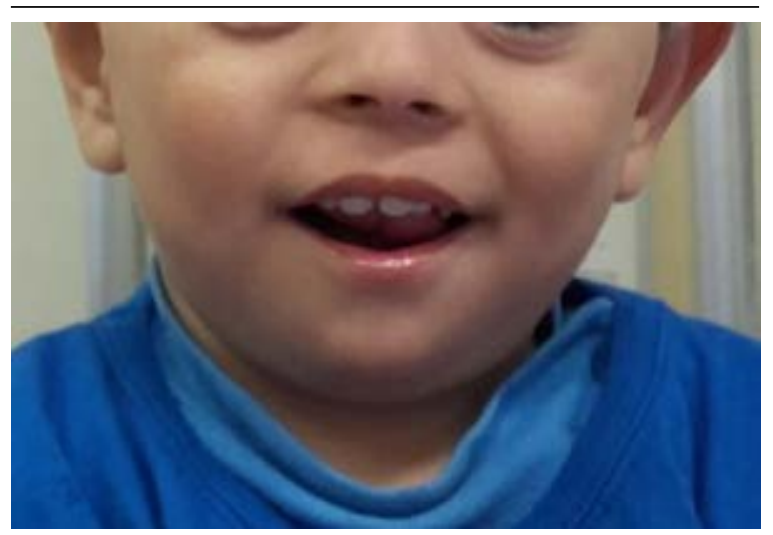

FIGURA 3. Opacidad corneal en el ojo izquierdo luego del tratamiento

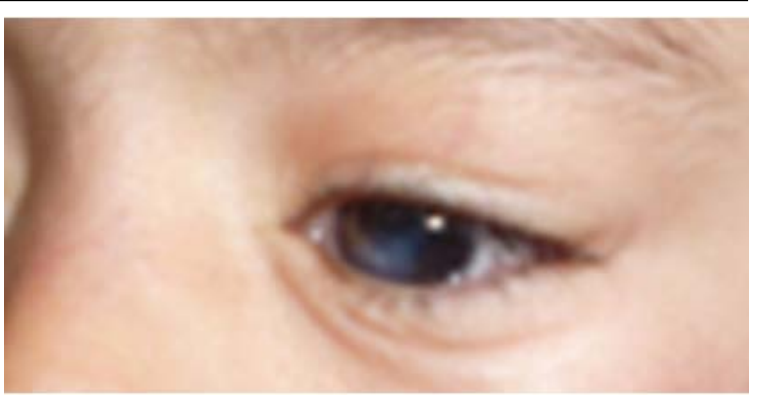


al diagnóstico oportuno. Miguel et al. reportaron a un paciente con el antecedente de 7 años de diagnósticos erróneos relacionados con la rosácea ocular. ${ }^{9}$

El abordaje terapéutico depende de la gravedad de la enfermedad. Todos los pacientes deben evitar los factores desencadenantes (temperaturas extremas, bebidas calientes, alimentos picantes, agentes tópicos irritantes, ingesta de alcohol y cigarrillos) y se debe aconsejar el uso de protector solar diario. Los pacientes con enfermedad cutánea de leve a moderada pueden realizar un tratamiento tópico (metronidazol, ivermectina, ácido azelaico, sulfacetamida sódica, retinoides). ${ }^{7} \mathrm{El}$ tratamiento de la rosácea ocular leve (blefaritis y conjuntivitis sin compromiso corneal) se basa en la higiene regular de los ojos y el uso de lágrimas artificiales. La blefaritis persistente, la neovascularización corneal y la infiltración marginal, así como el compromiso cutáneo grave, requieren, además de colirios oftálmicos, de corticoides y antibióticos tópicos (aminoglucósidos, macrólidos, quinolonas), tratamiento sistémico con una duración no menor de 6 meses. En los pacientes mayores de 8 años, se recomienda el uso de doxiciclina o minociclina a razón de $50 \mathrm{mg}$ dos veces al día. Debido a que la utilización de tetraciclinas está contraindicada en los menores de 8 años (pueden producir decoloración del esmalte dental, detención del crecimiento y del desarrollo óseo), los pacientes que requieran tratamiento antibiótico sistémico deben recibir macrólidos. La eritromicina utilizada en dosis de $30-50 \mathrm{mg} /$ $\mathrm{kg}$ / día dos veces al día durante 6 meses demostró ser una terapéutica eficaz en la afectación ocular y cutánea en este grupo etario. ${ }^{8,10}$ Las recurrencias son más frecuentes si el tratamiento se suspende rápidamente.

Gonser et al. realizaron un estudio de 19 niños con rosácea ocular que requirieron tratamiento sistémico y encontraron una tasa de remisión y recaída más baja que en los adultos luego de un tratamiento exitoso. Además, destacaron una mayor frecuencia de complicaciones graves y afectación corneal en este grupo etario. ${ }^{11}$
Los esteroides tópicos están indicados en los pacientes con queratitis o cicatrices y la ciclosporina tópica puede ser una alternativa útil si es necesario un tratamiento de mantenimiento antiinflamatorio. $^{8}$

\section{CONCLUSIÓN}

La rosácea es una enfermedad de presentación infrecuente en la edad pediátrica. Los síntomas y signos son inespecíficos, por lo cual se debe tener un alto índice de sospecha. La afectación ocular puede preceder a las manifestaciones cutáneas y demorar el diagnóstico. En el caso presentado, la corta edad del paciente y la falta de otras manifestaciones cutáneas, como el flushing y las telangiectasias, retrasaron el diagnóstico. Debe tenerse en cuenta que, ante la presencia de rosácea cutánea, es imprescindible la evaluación oftalmológica y el seguimiento del paciente en forma conjunta.

\section{REFERENCIAS}

1. Tan J, Berg M. Rosacea: current state of epidemiology. J Am Acad Dermatol. 2013; 69(6 Suppl 1):S27-35.

2. Lacz NL, Schwartz RA. Rosacea in the pediatric population. Cutis. 2004; 74(2):99-103.

3. Kellen R, Silverberg NB. Pediatric rosacea. Cutis. 2016; 98(1):49-53.

4. Chamaillard M, Mortemousque B, Boralevi F, Marques da Costa C, et al. Cutaneous and ocular signs of chilhood rosacea. Arch Dermatol. 2008; 144(2):167-71.

5. Stone DU, Chodosh J. Ocular rosacea: an update on pathogenesis and therapy. Curr Opin Ophthalmol. 2004; 15(6):499-502.

6. Keshtcar-Jafari A, Akhyani M, Ehsani AH, Ghiasi M, et al. Correlation of the severity of cutaneous rosacea with ocular rosacea. Indian J Dermatol Venereol Leprol. 2009; 75(4):405-6.

7. Kaminsky A, Flórez White M, Piquero Martín J, Herane MI, et al. Informe del consenso Ibero-Latinoamericano 2016 sobre la clasificación clínica y terapéutica de la rosácea. Med Cutan Iber Lat Am. 2016; 44(1):6-10.

8. Potz-Biedermann C, Mehra T, Deuter C, Zierhut M, et al. Ophthalmic rosacea: case report in a child and treatment recommendations. Pediatr Dermatol. 2015; 32(4):522-5.

9. Miguel AI, Salgado MB, Lisboa MS, Henriques F, et al. Pediatric ocular rosacea: 2 cases. Eur J Ophthalmol. 2012; 22(4):664-6.

10. Donaldson KE, Karp CL, Dunbar MT. Evaluation and treatment of children with ocular rosacea. Cornea. 2007; 26(1):42-6.

11. Gonser LI, Gonser CE, Deuter C, Heister M, et al. Systemic therapy of ocular and cutaneous rosacea in children. J Eur Acad Dermatol Venereol. 2017;31(10):1732-8. 